\begin{abstract}
Artigo selecionado por avaliadores aos pares e apresentado na XXI Semana Acadêmica de Fisioterapia da UFSM, realizada de 8 a 11 de novembro de 2017 no Auditório Gulerpe.
\end{abstract}

\title{
Apoio Social a mulheres com câncer de mama
}

\section{Social Support in women with breast cancer}

\author{
Ana Paula Donato, Betina Pivetta Vizzotto, Melissa Medeiros Braz
}

\section{RESUMO}

O câncer de mama apresenta incidência elevada e acomete principalmente a população feminina. Mesmo nos dias atuais, com o avanço nos métodos de tratamento, essa doença provoca alterações biopsicossociais na vida da mulher, que poderá repercutir a curto e longo prazo nas relações sociais. Assim, este estudo teve como objetivo avaliar o apoio social de mulheres em tratamento para o câncer de mama. Trata-se de uma pesquisa descritiva e transversal com abordagem quantitativa com 24 mulheres que aguardavam a consulta de rotina no ambulatório de Mastologia ou o atendimento de Fisioterapia. Para a coleta de dados, foi utilizado um questionário que continha informações sociodemográficas e a Escala de Apoio Social. Verificou-se, através do escore total obtido pelo Apoio Social de 72,17 $\pm 5,56$,que as mulheres recebiam um excelente apoio social em todos os domínios, destacando-se o apoio material. A partir dos dados obtidos, as mulheres deste estudo estão assistidas em relação ao apoio social, fator este que contribui no tratamento e na reinserção nas suas atividades.

Descritores: Apoio Social. Mulheres. Neoplasias da mama.

\section{ABSTRACT}

\section{Abstract}

Breast cancer has a high incidence and mainly affects the female population. Even today, with advances in treatment methods, this disease causes biopsychosocial changes in women's lives, which may have repercussions in the short and long term in social relationships. Thus, this study aimed to evaluate the social support of women in treatment for breast cancer. This is a descriptive and cross-sectional study with a quantitative approach with 24 women who were waiting for routine consultation in the Mastology outpatient clinic or Physiotherapy care. For the data collection, a questionnaire containing sociodemographic information and the Social Support Scale was used. It was verified, through the total score obtained by the Social Support of $72,17 \pm 5,56$, that the women received excellent social support in all domains, highlighting the material support. From the data obtained, the women in this study are assisted in relation to social support, a factor that contributes to the treatment and reinsertion in their activities.

Descriptors: Social support. Women. Breast neoplasms. 


\section{INTRODUÇÃO}

O câncer de mama ocorre devido à reprodução acelerada e desordenada de células mamárias, geneticamente alteradas, as quais se alojam na mama e podem se deslocar para outros tecidos e órgãos ${ }^{1}$. É considerada a neoplasia mais comum entre as mulheres, sendo a estimativa para o Brasil, no ano de 2016 de 57.960 casos novos e para a região Sul de 10.970 casos. No estado do Rio Grande do Sul estimaram-se 5.210 novos casos para o mesmo ano².

Distintos são os fatores de risco associados ao desenvolvimento dessa neoplasia, entre eles os genéticos, ambientais, a idade, hábitos de vida, medicamentos e hereditariedade ${ }^{3}$.

A detecção precoce possibilita recursos menos mutiladores e maior chance de cura, no entanto o procedimento cirúrgico, em especial a mastectomia, ainda é o método mais utilizado para o controle da doença. A mastectomia é descrita por muitas mulheres como um momento traumatizante e agressivo, que pode interferir sobre o seu cotidiano e a sua imagem corporal, devido à mama tratar-se de um órgão com importante representação da feminilidade ${ }^{4}$.

Devido à pressão da sociedade, a mulher idealiza modelos de beleza e as mamas passam a serem vistas como símbolos, além de maternidade e feminilidade, também de sexualidade. Quando surge, devido ao câncer, a necessidade da retirada de uma ou das duas mamas, a percepção do corpo pelo cérebro é modificada, e com isso a imagem corporal necessita ser ajustada a uma nova situação ${ }^{5}$.

A retirada da mama provoca interferência negativa na autoestima, e um sentimento de angústia e estresse pode ser vivenciado por muitas mulheres ao estarem despidas diante de seus companheiros. Pode ocorrer também o medo da reação das pessoas na sociedade em observá-las sem a(s) mama(s), e diante desta realidade muitas mulheres usam roupas as quais possam disfarçar a ausência da(s) mesma(s)

Segundo Romeiro et al.7, a associação entre câncer de mama e apoio social tem sido descrita como um fator que contribui na recuperação e no tratamento das mulheres com essa neoplasia. Através da rede de apoio elas são encorajadas a enfrentar todas as difíceis fases da doença, reduzindo assim o impacto negativo provocado pela patologia em sua vida.

No entanto, os efeitos do apoio social exercidos sobre sistema imunológico permanecem obscuros, porém duas proposições são expostas. Uma, que o apoio social atuaria como "tampão", bloqueando a resposta do organismo em desenvolver uma patologia, e a outra, que poderia aumentar a percepção de controle sobre a sua própria vida, implicando em efeitos positivos sobre a saúde.

Desse modo, esta pesquisa teve como objetivo verificar o apoio social de mulheres em tratamento para o câncer de mama.

\section{METODOLOGIA}

O estudo é caracterizado como descritivo e transversal com abordagem quantitativa, foi realizado no período de janeiro a abril de 2017. A pesquisa teve início após aprovação do Comitê de Ética em Pesquisa (CEP) institucional na data de 28 de novembro de 2016, sob o número de parecer 1.838 .849 e obedece aos critérios estabelecidos pela resolução 466/12 no CNS.

A amostra foi constituída por mulheres com diagnóstico médico de câncer de mama. Foram incluídas na pesquisa mulheres, independente da faixa etária, que haviam realizado tratamento cirúrgico, radical ou conservador, com ou sem reconstrução mamária e que apresentavam ou não linfedema. Sendo excluídas as mulheres que estavam realizando tratamento quimioterápico e aquelas que não se declarassem em condições de responder à entrevista.

A população alvo foi convidada pela pesquisadora, pessoalmente, para que participasse de forma voluntária do estudo, sendo também prestados esclarecimentos sobre a pesquisa e seus objetivos. Aquelas que aceitaram assinaram 0 Termo de Consentimento Livre e Esclarecido em duas vias, sendo que uma delas permaneceu em posse da pesquisadora.

Posteriormente, foram aplicados os questionários, de forma individual, em uma sala reservada, enquanto elas aguardavam a consulta de rotina ou o atendimento fisioterapêutico no Hospital Universitário, no ambulatório de Fisioterapia e no Centro de Referência da Saúde do Trabalhador (CEREST) da cidade onde foi realizada a pesquisa.

Foi utilizada umaficha de identificação adaptada de Pivetta et al.9, continha informações sociodemográficas (idade, estado civil, cor, $n^{0}$ de filhos) das voluntárias. A fim de coletar os dados sobre 0 apoio social, foi utilizado 0 questionário proposto pelo Medical OutcomesStudy (MOS)10 e validado para o português por Griep et al.11. Trata-se 
de uma escala composta por 19 itens, compreendendo cinco dimensões funcionais: material (4 perguntas), afetivo (3 perguntas), emocional (4 perguntas), interação social positiva (4 perguntas) e informação (4 perguntas). Para todos os itens, cinco opções de resposta, variando de "nunca" a "sempre", são apresentadas. Na análise, a escala varia de 0 a 4 , sendo utilizado o somatório das respostas, cujo resultado poderá variar de 0 a 76; quanto maior o escore, maior 0 apoio social.

A aplicação dos instrumentos de avaliação foram realizados apenas pela pesquisadora. Para a análise dos dados, foi realizada a digitação no programa Excel 2003 para armazenamento. Inicialmente foram realizadas análises univariadas para caracterização da amostra. Para as variáveis contínuas foi realizado o teste de normalidade de Shapiro-Wilk.

\section{RESULTADOS}

Foram aplicados 31 questionários, sendo 7 excluídos pois não se encaixavam nos critérios de inclusão do estudo, restando pela elegibilidade 24 .

Na tabela I, apresentam-se os dados de caracterização das mulheres, das quais 62,50\% declararam-se ser de cor branca, $62,50 \%$ eram casadas ou estavam em coabitação com o companheiro.

Tabela I - Dados de caracterização das mulheres em tratamento para o câncer de mama $(n=24)$.

\begin{tabular}{llll}
\hline \multicolumn{1}{c}{ VARIÁVEIS } & N & $\%$ & MÉDIA E DP \\
\hline $\begin{array}{l}\text { Idade no momento do } \\
\text { diagnóstico }\end{array}$ & 24 & & $47,79 \pm 7,49$ \\
\hline
\end{tabular}

\begin{tabular}{lcc}
\hline Cor & 15 & 62,50 \\
Branca & 5 & 20,83 \\
Negra & 2 & 8,33 \\
Parda & 2 & 8,33 \\
Não informado & & \\
\hline & & \\
Estado Civil & 15 & 62,50 \\
Casada ou coabitação & 3 & 12,50 \\
Solteira & 3 & 12,50 \\
Viúva & 3 & 12,50 \\
Não informado & & \\
\hline & & \\
No de filhos & 3 & 12,50 \\
Nenhum & 7 & 29,17 \\
1 & 6 & 25,00 \\
2 & 5 & 20,83 \\
3 & 3 & 12,50 \\
Mais de 3 & & \\
\hline
\end{tabular}

Na tabela II, estão apresentados os domínios e escore total do apoio social das mulheres que fizeram parte desta pesquisa, representados por meio da média e desvio padrão.

A média do escore total do apoio social aproximou-se do valor máximo de 76 pontos, o que demonstra que as mulheres avaliavam receber ótimo apoio social em todos os domínios, destacando-se o apoio material com 15,46 pontos. 
Tabela II- Apoio social de mulheres em tratamento para o câncer de mama, apresentados em média e desvio padrão, por meio dos domínios e escore total do MOS.

\begin{tabular}{lc}
\hline Apoio Social (MOS) & Escores (média \pm DP) \\
\hline Apoio Material & $15,46 \pm 1,14$ \\
\hline Apoio Afetivo & $11,79 \pm 0,51$ \\
\hline Apoio Emocional & $15,25 \pm 1,92$ \\
\hline Apoio de Informação & $15,29 \pm 1,43$ \\
\hline Interação Social Positiva & $14,46 \pm 2,75$ \\
\hline Total & $72,17 \pm 5,56$ \\
\hline
\end{tabular}

\section{DISCUSSÃO}

A partir da visão e da abordagem que o fisioterapeuta apresenta em relação ao paciente, procurando não tratar somente das necessidades físicas, mas sim compreender o contexto social em que pode estar interferindo no sucesso da terapêutica, o presente estudo explorou o apoio social de 24 mulheres em tratamento para o câncer de mama.

Entre as características sociodemográficas analisadas, a idade média das mulheres no momento do diagnóstico foi de 47,79 anos. Estudos apontam que esta patologia é rara antes dos 35 anos, no entanto sua incidência passa a ser rápida, crescente e progressiva a partir dessa idade, indicando que após os 50 anos quatro em cada cinco casos ocorre ${ }^{12-13}$

A cor/raça de maior predominância neste estudo foi a branca, esse dado corrobora ao estudo de Nunes et al.14, em que das 101 mulheres diagnosticadas com câncer de mama, houve maior predominância desta neoplasia das pacientes da cor branca $(44,4 \%)$, seguida pela cor parda $(39,1 \%)$ e pela cor negra $(14,5 \%)$.

A maioria delas $(62,5 \%)$ eram casadas e $87,5 \%$ tinham entre 1 ou mais de 3 filhos, sendo este dado considerado positivo, pois o companheiro ou o familiar é considerado como fator que contribuem no enfrentamento da doença. Ferreira et al.4, abordam que o apoio oferecido pelo esposo para sua parceira faz com que a mesma vivencie esta experiência da doença de forma menos traumática e atemorizante. Diferentemente ocorre naquelas mulheres que não recebem o apoio, estas se sentem mais fragilizadas o que interfere de maneira negativa na luta contra o câncer.

$\mathrm{Na}$ avaliação do apoio social, as mulheres avaliaram receber excelente apoio social em todos os domínios, destacando-se o apoio material, que se refere a recursos financeiros e material em que são oferecidos a outras pessoas em situação de necessidade por que passa o sujeito. Sendo assim, neste estudo, sugere-se que as mulheres recebiam e estavam satisfeitas com o auxílio no cuidado das suas atividades de vida diária, seja com os filhos, casa ou com outras demandas desta ordem, enquanto esta encontra-se com dificuldades física e emocionais ${ }^{15}$.

Outro domínio com resultado positivo foi o apoio de informação, o qual faz menção a sugestões, orientações prestadas inclusive pelos profissionais da saúde. Ambrósio, Santos16, abordam sobre a necessidade e importância dos profissionais da saúde, estarem atentos e sensíveis as necessidade e demandas individuais, não somente físicas, mas sim ao psíquico e social, com o intuito de prestar assistência adequada e efetiva a essas mulheres. Além disso, hoje os grupos de apoio estão vinculados a recursos que auxiliam no enfrentamento e na superação da doença, uma vez que a socialização promove trocas de experiências que contribui na luta contra a patologia. 


\section{CONCLUSÃO}

A partir dos dados obtidos, constatou-se que as mulheres deste estudo estão assistidas em relação ao apoio social, fator este que contribui no enfrentamento da doença e na superação das difíceis fases do tratamento, além de colaborar na reinserção desta mulher em suas atividades e em seus papeis até então desempenhados.

\section{REFERÊNCIAS}

1 Furlan ALA; Neto MS; Abla LEF; Oliveira CJR; Lima AC; Ruiz BFO et al. Qualidade de vida e autoestima de pacientes mastectomizadas submetidas ou não a reconstrução de mama. ReviBrasCir Plást. 2013; 28(2):9-264. [http:// dx.doi.org/10.1590/S1983-51752013000200016].

2 Instituto Nacional de Câncer José Alencar Gomes da Silva. Disponível em: http://www2.inca.gov.br/wps/wcm/ connect/acoes_programas/site/home/nobrasil/programa_controle_cancer_mama/conceito_magnitude. Acesso em:16 de julho de 2017

3 Pinheiro AB; Lauter DS; Medeiros GC; Cardozo IR; Menezes LM; Souza RMB et al. Câncer de Mama em Mulheres Jovens: Análise de 12.689 Casos.Rev Bras Cancerol. 2013; 59(3):352-359.

4 Ferreira DB; Faragol PM; Reis PED; Funchetto SS. Nossa vida após o câncer de mama: percepções e repercussões sob o olhar do casal. Rev Bras Enferm. 2011; 64(3):44-536. [http://dx.doi.org/10.1590/S0034-71672011000300018].

5 Ciacco M; Rezende LF. Avaliação da imagem corporal em mulheres no pós-operatório de câncer de mama. Rev Bras Mastol.2012; 22(4):131-137.

6 Prates ACL; Junior RF; Prates MFO; D`Alessandro AAB; Veloso MF. Indicadores de insatisfação relacionados à imagem corporal em pacientes submetidas à mastectomia. Rev Bras Mastol. 2014; 24(1):8-23. [doi: 10.5327/ Z201400010005RBM].

7 Romeiro FB; Both LM; Machado ACA; Lawrenz P; Castro EK. O Apoio Social das Mulheres com Câncer de Mama: Revisão de Artigos Científicos Brasileiros. Rev Psicol Saude. 2012; 4(1):27-38. [doi: http://dx.doi.org/10.20435/ pssa.v4i1.122].

8 Amaral FLJS; Guerra RO; Nascimento AFF; Maciel ACC. Apoio social e síndrome da fragilidade em idosos residentes na comunidade.Ciênc Saúde Colet. 2013, 18(6):1835-1846. [doi: http://dx.doi.org/10.1590/S141381232013000600034].

9 Pivetta HMP, Braz MM Petter GNP, Segala M, Jobim FC, Martins, TNO et al. Prevalência de fatores de risco de mulheres com câncer de mama. Rev.Ciênc. Méd. Biol. 2014;13(2):170-175. [doi:10.9771/cmbio.v13i2.12134].

10 Sherbourne DD; Stewrt AL. The MOS Social Support Survey. Social Science and Medicine.1991;38(6):705-714.

11 Griep RH; Chor D; Faerstein E; Werneck GL; Lopes CS.Validade de constructo de escala de apoio social do Medical Outcomes Study adaptada para o português no Estudo Pró-Saúde.Cad. Saúde Pública. 2005, 21(3):14-703. [doi:http://dx.doi.org/10.1590/S0102-311X2005000300004].

12 Ribeiro VC; Portella SDC; Malheiros ES. Mulheres de meia idade e o enfrentamento do câncer de mama.Rev Cuid. 2014; 5(2):700-805. [doi:http://dx.doi.org/10.15649/cuidarte.v5i2.120]. 
13 Souza CB; Fustinoni SM; Amorim MH; Zandonade E; Matos JC; Schirmer J. Estudo do tempo do diagnóstico e início do tratamento do câncer de mama em idosas de um hospital de referência em São Paulo, Brasil. Ciênc Saúde Colet. 2015; 20(12):3805-3816. [doi:10.1590/1413-12320152012.00422015].

14 Nunes, BAP; Siqueira, SL; Pereira, SM; Pacheco, TJ; Pessanha TO; Mendonça SB. Perfil epidemiológico dos pacientes diagnosticados com câncer de mama em Campos dos Goytacazes (RJ), Brasil. Rev Bras Mastol. 2012; 22(4):117-123.

15 Almeida TG; Cocassetto I; Alves KM; Santos AAP; Silva JMO; Trezza MCSF. Vivência da mulher jovem com câncer de mama e mastectomizada.Esc Anna Nery. 2015; 9(3):432-438.[doi:10.5935/1414-8145.20150057].

16 Ambrósio DCM; Santos MA. Apoio social à mulher mastectomizada: um estudo de revisão.Ciênc Saúde Colet. 2015; 20(3):851-864. [doi:10.1590/1413-81232015203.13482014]. 